Research Article

\title{
Serum ICAM-1 as a Predictor of Prognosis in Patients with Acute Ischemic Stroke
}

\author{
Lei Wang, ${ }^{1}$ Yan Chen, ${ }^{2}$ Depeng Feng, ${ }^{3}$ and Xiaoling Wang $\mathbb{D}^{3}$ \\ ${ }^{1}$ Department of Infectious Disease, Beijing Tiantan Hospital, Capital Medical University, Beijing, China \\ ${ }^{2}$ Department of Geriatric Medicine, Shandong Provincial Third Hospital, Cheeloo College of Medicine, Shandong University, Jinan, \\ Shandong Province, China \\ ${ }^{3}$ Department of Neurology, Liaocheng People's Hospital, Liaocheng, Shandong Province, China
}

Correspondence should be addressed to Xiaoling Wang; haiyun900@163.com

Received 17 February 2021; Revised 9 March 2021; Accepted 11 March 2021; Published 19 March 2021

Academic Editor: Yuzhen Xu

Copyright ( 92021 Lei Wang et al. This is an open access article distributed under the Creative Commons Attribution License, which permits unrestricted use, distribution, and reproduction in any medium, provided the original work is properly cited.

\begin{abstract}
Objective. Inflammation is one of the key mechanisms involved in functional impairment after stroke. Intercellular adhesion molecule-1 (ICAM-1) is an important inflammatory molecule in the body. The purpose of our study was to determine the correlation between ICAM-1 and the prognosis of acute ischemic stroke (AIS). Methods. 286 AIS patients treated at Beijing Tiantan Hospital were continuously included in the study. The demographic data of the patients were collected, and the fasting blood within 24 hours of admission was collected to detect the clinical indicators. The functional prognosis was measured using the modified Rankin Scale (mRS) 3 months after stroke. The poor prognosis is defined as mRS $\geq 3$. The enzyme-linked immunosorbent assay (ELISA) was used to determine the serum ICAM-1 levels. Results. The serum ICAM-1 levels of patients with poor prognosis were significantly higher than that of patients with good prognosis $(144.2 \pm 14.8 \mathrm{vs} 117.5 \pm 12.1 \mathrm{pg} / \mathrm{ml})$. Receiver operating characteristic curve (ROC) analysis showed that the sensitivity and specificity of serum ICAM-1 for predicting the prognosis of AIS were $74 \%$ and $76 \%$, respectively. In logistic regression analysis, the serum ICAM-1 level is still an independent predictor of poor prognosis (odds ratio [OR]: 0.52; 95\% confidence interval [CI]: 0.318-0.839). Conclusions. Higher serum ICAM-1 levels on admission in AIS patients might increase the risk of poor prognosis.
\end{abstract}

\section{Introduction}

The definition of stroke is a neurological impairment syndrome caused by the permanent brain, spinal cord, or retinal cell death caused by vascular etiology [1]. The morbidity and disability rate of stroke are relatively high, with 13.7 million new stroke patients every year, and 5.8 million deaths due to stroke [2]. In the world, more than 80 million people have survived strokes, of which about $70 \%$ are ischemic strokes, and the rest are cerebral hemorrhage ( $\mathrm{ICH})$ or subarachnoid hemorrhage (SAH). Stroke is the second leading cause of death in the world, while it ranks first in China. China's national research and regular government reports show that the burden of stroke is high and is gradually increasing [3]. The pathogenic mechanism of AIS is complicated, and there is no cure $[4,5]$. Therefore, finding the pathogenic target of AIS and developing targeted treatment are urgent problems to be solved.

Intercellular adhesion molecule-1 (ICAM-1), also named $\mathrm{CD} 54$, is a type I transmembrane protein with a molecular weight between 80 and $114 \mathrm{kDa}$, and its molecular weight varies depending on the level of glycosylation [6]. The full length of ICAM-1 consists of five immunoglobulin (Ig) domains, a short cytoplasmic tail with multiple threonine residues, and a transmembrane domain [7]. Alternative splicing is a common post transcriptional mechanism that is widely used to regulate the gene expression [8]. ICAM-1 can produce soluble ICAM-1 through alternative splicing, which can be measured in various body fluids [9]. Studies have shown that ICAM-1 levels are elevated in atherosclerosis, cardiovascular 
disease, and metabolic vascular neuropathy [10-13]. However, there are still few studies on the correlation between ICAM-1 and AIS.

Neurons, microglia, astrocytes, and vascular components together form a functional neurovascular unit, which is the anatomical basis for the pathogenesis of AIS [14, 15]. Microglia are important innate immune cells in the body, and their different activation states exert different inflammatory effects [16-18]. More and more evidences show that inflammation plays a key role in the pathogenesis of AIS and may become a potential target for treatment $[19,20]$. In this study, we explored the predictive effect of the inflammatory molecule ICAM-1 on the prognosis of AIS, which may provide an important target for the therapeutic intervention of stroke.

\section{Methods}

2.1. Study Population. From January 2020 to December 2020, AIS patients who were treated at Beijing Tiantan Hospital were included in the study. After screening, a total of 286 patients were included in the study. An AIS attack is defined as the patient's first neurological deficit, which is confirmed by brain CT or MRI. The exclusion criteria include (1) past history of stroke, (2) combined with tumor, (3) combined with infectious disease or autoimmune disease, (4) intravenous thrombolysis or arterial thrombectomy, and (5) combined with cerebral hemorrhage. The study complied with the Declaration of Helsinki and was approved by the local medical ethics committee. Research subjects or guardians agree to participate in the research and sign an informed consent form.

2.2. Characteristics of AIS Patients. All subjects underwent the National Institute of Health Stroke Scale immediately after enrollment to evaluate the patients' neurological function. Their demographic data (age and gender) were collected. At the same time, the past medical history (hypertension, diabetes, coronary heart disease, and atrial fibrillation) was asked and recorded. An electronic sphygmomanometer is used to measure the patient's blood pressure in a quiet state. The patient's fasting blood glucose and total cholesterol were measured in the biochemical laboratory.

2.3. Serum ICAM-1 Determination. Fasting blood of all patients was collected within 24 hours of admission. Peripheral blood was allowed to stand for half an hour at room temperature and then centrifuged at low temperature and high speed. Separate and pack the serum and store it in a refrigerator at $-80^{\circ} \mathrm{C}$ for later use. The concentration of serum ICAM-1 was determined by the enzyme-linked immunosorbent assay (ELISA). All ICAM-1 antibodies for the experiment were purchased from Abcam Co., Ltd. (Abcam, Cambridge, MA). The ELISA test method refers to the previous literature and product instructions [21-23].

2.4. Prognosis Assessment and Grouping. The modified Rankin Scale (mRS) is a commonly used neuroevaluation scale, mainly used to evaluate the degree of disability in patients with neurological dysfunction. The scale was first proposed by Dr. John Rankin of Scotland in 1957, and it has become the most widely used clinical outcome tool in stroke clinical trials [24-26]. In this study, the modified Rankin Score (mRS) was used to measure the functional prognosis 3 months after stroke. A good prognosis is defined as $\mathrm{mRS}<$ 3 , and a poor prognosis is defined as $m R S \geq 3$. According to the difference of mRS scores, AIS patients were divided into the good outcome group and poor outcome group.

2.5. Statistical Analysis. Continuous variables were described as mean \pm standard deviation or median and interquartile range and compared with Student's $t$-test. Categorical variables are described as $n$ (\%) and compared with the chisquare test. The receiver operating characteristic curve is applied to determine the cut point of ICAM-1 to distinguish between good and poor outcomes. Logistic regression analysis was used to evaluate the independent contribution of different variables to prognosis prediction. Statistical analysis was performed using SPSS22.0 software (SPSS Inc., Chicago, Illinois, USA), and $p$ value $<0.05$ was considered statistically significant.

\section{Results}

3.1. Characteristics of AIS Patients. A total of 286 AIS patients were included in this study. According to the mRS score, they were divided into a good outcome group $(n=181)$ and a poor outcome group $(n=105)$. The ages of AIS patients in the good outcome group and poor outcome group were $(61.4 \pm 8.9)$ and $(61.7 \pm 9.6)$ years, respectively. The proportion of men with AIS in the good outcome group and poor outcome group was $60.8 \%$ and $59.0 \%$, respectively. There was no significant statistical difference in age and gender between the two groups $(p>0.05)$. We further compared the past medical history of the two groups of AIS patients, and we found that their incidence of hypertension, diabetes, coronary heart disease, and atrial fibrillation was not significantly different $(p>0.05)$. Regarding clinical indicators, such as systolic blood pressure, diastolic blood pressure, fasting blood glucose, and total cholesterol, there was also no significant statistical difference between the two groups $(p>0.05)$. However, the serum ICAM-1 concentrations of AIS patients in the good outcome group and the poor outcome group were $(117.5 \pm 12.1)$ and $(144.2 \pm 14.8) \mathrm{pg} / \mathrm{ml}$, respectively, and the serum ICAM-2 levels of AIS patients in the poor outcome group were significantly higher in the good outcome group $(p<0.001)$. The characteristics of all AIS patients are shown in Table 1.

3.2. ROC Analysis for the Serum ICAM-1 Level in the AIS Patients. As shown in Figure 1, in order to evaluate the diagnostic value of serum ICAM-1 as a potential prognostic marker of AIS, we performed ROC curve analysis. For serum ICAM-1, the sensitivity is $74 \%$, the specificity is $76 \%$, and the area under the curve (AUC) is 0.772 . The best cutoff value of the ROC curve for distinguishing good outcomes from poor outcomes is $129.5 \mathrm{pg} / \mathrm{ml}$. When the baseline serum ICAM-1 concentration is higher than $129.5 \mathrm{pg} / \mathrm{ml}$, it indicates that the prognosis of AIS patients is poor. However, when the 
TABLE 1: Characteristics of AIS patients with poor outcome and good outcome.

\begin{tabular}{|c|c|c|c|}
\hline & Good outcome $(n=181)$ & Poor outcome $(n=105)$ & $p$ value \\
\hline Age, years & $61.4 \pm 8.9$ & $61.7 \pm 9.6$ & 0.790 \\
\hline Male, $n(\%)$ & $110(60.8)$ & $62(59.0)$ & 0.774 \\
\hline NHISS on admission & $5(2-7)$ & $10(8-14)$ & $<0.001$ \\
\hline Hypertension, $n(\%)$ & $89(49.2)$ & $53(50.5)$ & 0.832 \\
\hline Diabetes mellitus, $n(\%)$ & $54(29.8)$ & $32(30.5)$ & 0.909 \\
\hline Coronary heart disease, $n(\%)$ & $19(10.5)$ & $12(11.4)$ & 0.807 \\
\hline Atrial fibrillation, $n(\%)$ & $10(5.5)$ & $6(5.7)$ & 0.946 \\
\hline Systolic blood pressure, $\mathrm{mmHg}$ & $148.3 \pm 10.2$ & $148.5 \pm 10.7$ & 0.875 \\
\hline Diastolic blood pressure, $\mathrm{mmHg}$ & $92.6 \pm 7.9$ & $92.8 \pm 8.3$ & 0.840 \\
\hline Fasting blood-glucose, mmol/l & $6.2 \pm 0.8$ & $6.3 \pm 0.9$ & 0.332 \\
\hline Total cholesterol, mmol/l & $4.7 \pm 1.2$ & $4.8 \pm 1.1$ & 0.484 \\
\hline ICAM-1, pg/ml & $117.5 \pm 12.1$ & $144.2 \pm 14.8$ & $<0.001$ \\
\hline
\end{tabular}

AIS: acute ischemic stroke; NIHSS: National Institute of Health Stroke Scale; ICAM-1: intercellular adhesion molecule-1.

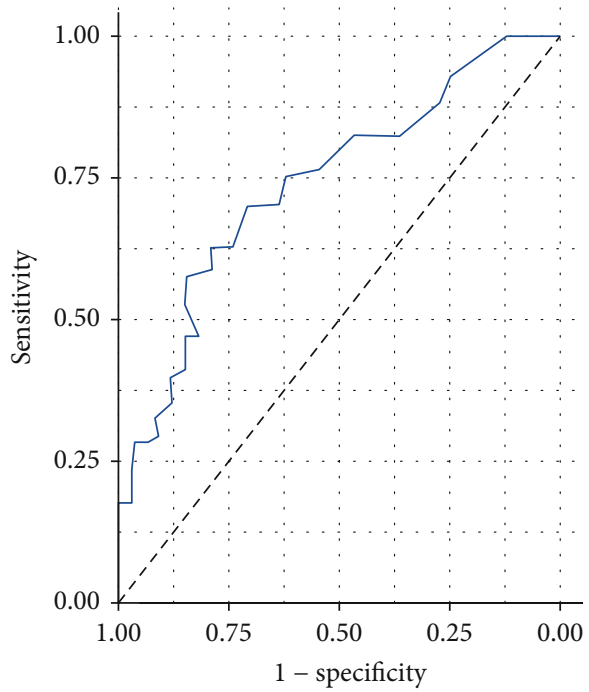

Figure 1: ROC analysis for the serum ICAM-1 level in the AIS patients.

baseline serum ICAM-1 concentration is lower than $129.5 \mathrm{pg} / \mathrm{ml}$, it indicates that AIS patients have a good prognosis.

3.3. Logistic Regression Analysis. The clinical baseline characteristics were incorporated into logistic regression to analyze the predictive value of different variables on the prognosis of AIS. Clinical baseline characteristics include age, gender, NHISS score, hypertension, coronary heart disease, diabetes, atrial fibrillation, systolic blood pressure, diastolic blood pressure, fasting blood glucose, total cholesterol, and serum ICAM-1 concentration. The results of the logistic regression analysis are shown in Table 2. The results showed that after adjusting for age, gender, NHISS score, hypertension, coronary heart disease, diabetes, atrial fibrillation, systolic blood pressure, diastolic blood pressure, fasting blood glucose,
TABle 2: Risks variables for poor outcome at 3 months in AIS patients.

\begin{tabular}{lccc}
\hline & OR & $95 \%$ CI & $p$ value \\
\hline Age, years & 1.35 & $0.843-1.418$ & 0.231 \\
Male, $n$ (\%) & 1.16 & $0.902-1.194$ & 0.365 \\
NHISS on admission & 0.65 & $0.436-0.803$ & 0.058 \\
Hypertension, $n$ (\%) & 1.33 & $0.887-1.379$ & 0.272 \\
Diabetes mellitus, $n(\%)$ & 1.31 & $0.835-1.352$ & 0.107 \\
Coronary heart disease, $n(\%)$ & 1.18 & $0.891-1.205$ & 0.329 \\
Atrial fibrillation, $n(\%)$ & 1.24 & $0.858-1.287$ & 0.353 \\
Systolic blood pressure, mmHg & 1.35 & $0.774-1.386$ & 0.432 \\
Diastolic blood pressure, mmHg & 1.31 & $0.970-1.361$ & 0.504 \\
Fasting blood-glucose, mmol/l & 1.27 & $0.859-1.304$ & 0.766 \\
Total cholesterol, mmol/l & 1.20 & $0.831-1.275$ & 0.520 \\
ICAM-1, pg/ml & 0.52 & $0.318-0.839$ & 0.007 \\
\hline
\end{tabular}

AIS: acute ischemic stroke; NIHSS: National Institute of Health Stroke Scale; ICAM-1: intercellular adhesion molecule-1.

and total cholesterol, the serum ICAM-1 level is still the prognosis of AIS patients' independent predictor $(p=0.007)$.

\section{Discussion}

The main finding of our current study is that the serum ICAM-1 levels of patients with poor AIS outcomes are significantly higher than those with good outcomes. Our further ROC analysis indicated that the cut-off value of serum ICAM-1 as a diagnostic target for predicting the prognosis of AIS was $129.5 \mathrm{pg} / \mathrm{ml}$, and its sensitivity and specificity were $74 \%$ and $76 \%$, respectively. After adjusting the clinical baseline data, the serum ICAM-1 level can still be used as an independent biomarker for predicting the prognosis of AIS patients. 
Inflammation is the main factor affecting the pathobiology and prognosis of AIS [27]. Although the inflammatory response begins with local occlusion and hypoperfusion of blood vessels and ischemic brain parenchyma, the inflammatory mediators generated in situ spread throughout the organism, leading to systemic inflammation [28]. Increased blood levels of systemic inflammatory markers are associated with adverse outcomes after AIS. Animal studies have demonstrated the causal relationship between systemic inflammation and AIS neurological damage and poor prognosis [29]. The underlying mechanisms of systemic inflammation affecting the poor prognosis of AIS include neutrophil infiltration, blood-brain barrier (BBB) destruction, and cerebral ischemia-reperfusion injury [30, 31]. Animal experiments showed that reducing leukocyte infiltration could reduce the neurological damage of AIS and delay disease progression [32]. However, clinical trials of antileukocyte therapy have not reached a consistent conclusion with animal experiments.

ICAM-1 is a glycoprotein adhesion receptor mainly expressed on the surface of endothelial cells $[33,34]$. Its main function is similar to chemokines and can induce the recruitment of leukocytes from the circulation to the inflammation site [35]. The unique domain of ICAM-1 allows it to be used as a biosensor, which can associate with the actin cytoskeleton after binding to a ligand to achieve signal transduction [36]. sICAM-1 is produced as a splice isoform of ICAM-1 or as a result of proteolytic cleavage. sICAM-1 retains all 5 extracellular Ig domains, and the molecular composition of the Ig domain of sICAM-1 varies depending on the protease that catalyzes the cleavage [9]. Studies have found that sICAM-1 has the dual effects of proinflammatory and antiinflammatory [37], which suggests the complexity of its involvement in pathogenic mechanisms.

The mechanism of ICAM-1 involved in neuroinflammatory injury after stroke has been reported. Research by Zhang et al. showed that anti-ICAM-1 antibody could significantly reduce ischemic brain damage after focal cerebral ischemia in rats, while reducing polymorphonuclear leukocytes in the injured area [38]. Consistent with the above results, Bowes et al. further verified that anti-ICAM-1 could reduce neurological damage after stroke in a rabbit cerebral ischemia model, but it had no synergistic effect with tissue plasminogen activator (tPA) [39]. However, there are also different conclusions in animal experiments. Studies have shown that ICAM-1 knockout mice can neither inhibit the recruitment of neutrophils after cerebral ischemia nor can they provide protection for neurons after ischemia and reperfusion [40]. These different conclusions precisely suggest that ICAM-1 is involved in the complexity of inflammatory damage after stroke.

Not only in animal experiments but also in clinical studies, ICAM-1 has also been shown to be associated with ischemic stroke. A study in Finland showed that the expression of ICAM-1 was significantly upregulated after AIS, and it worked in concert with chemokines to cause granulocyte infiltration. This may be one of the potential mechanisms of ICAM-1 aggravating the neurological damage in stroke [41]. Unlike the above study, a clinical study in Croatia showed that serum ICAM-1 levels have nothing to do with the severity of stroke [42]. The explanation for this completely different conclusions may be as follows: first, the difference of race and second, the Croatian study is grouped according to different stroke subtypes, which may be the main reason for the different conclusions from the Finnish study. The results of Enlimomab Acute Stroke Trial Investigators showed that anti-ICAM-1 treatment not only failed to reduce the neurological damage of ischemic stroke but also significantly worsened the stroke outcome [43]. Interestingly, a recent study from China showed that serum ICAM-1 levels in AIS patients were elevated, and it was related to the increased risk of hemorrhage conversion after AIS [44].

Our research has some limitations. Firstly, our AIS patients come from a single center, and the sample size is small. Secondly, we have not dynamically monitored the changes in serum ICAM-1 nor have we conducted longterm follow-up of AIS patients. Thirdly, our AIS patients do not include patients treated with recombinant plasminogen activator (rtPA) and healthy volunteers. Therefore, we cannot evaluate the correlation between ICAM-1 and prognosis in patients with AIS after intravenous thrombolysis. Fourthly, we did not detect the levels of serum traditional inflammation markers nor did we explore their correlation with ICAM-1. Finally, we did not perform subgroup analysis of AIS patients with different causes. However, our research still has obvious advantages. Our study reported for the first time the correlation between serum ICAM-1 levels and the short-term prognosis of AIS stroke.

\section{Conclusions}

The level of serum ICAM-1 in AIS patients with poor prognosis was significantly higher than that in the good prognosis group. The baseline serum ICAM-1 level predicts the prognosis of AIS with high sensitivity and specificity. And after adjusting for multivariate, the serum ICAM-1 level is still an independent predictor of AIS functional outcome. The study of stroke targeting ICAM-1 is currently controversial, and more studies are still needed in the future to clarify this complex pathological mechanism.

\section{Data Availability}

The data used to support the findings of this study are available from the corresponding author upon request.

\section{Conflicts of Interest}

All the authors declare no conflict of interests.

\section{References}

[1] Y. Xu, K. Wang, Q. Wang, Y. Ma, and X. Liu, "The antioxidant enzyme PON1: a potential prognostic predictor of acute ischemic stroke," Oxidative Medicine and Cellular Longevity, vol. 2021, Article ID 6677111, 8 pages, 2021.

[2] M. S. Phipps and C. A. Cronin, "Management of acute ischemic stroke," BMJ, vol. 368, p. 16983, 2020. 
[3] S. Wu, B. Wu, M. Liu et al., "Stroke in China: advances and challenges in epidemiology, prevention, and management," Lancet Neurology, vol. 18, no. 4, pp. 394-405, 2019.

[4] Y. Xu, Q. Wang, J. Chen, Y. Ma, and X. Liu, "Updating a strategy for histone deacetylases and its inhibitors in the potential treatment of cerebral ischemic stroke," Disease Markers, vol. 2020, Article ID 8820803, 8 pages, 2020.

[5] T. Leng and Z. G. Xiong, "Treatment for ischemic stroke: from thrombolysis to thrombectomy and remaining challenges," Brain Circ, vol. 5, no. 1, pp. 8-11, 2019.

[6] C. Lawson and S. Wolf, "ICAM-1 signaling in endothelial cells," Pharmacological Reports, vol. 61, no. 1, pp. 22-32, 2009.

[7] T. N. Ramos, D. C. Bullard, and S. R. Barnum, "ICAM-1: isoforms and phenotypes," Journal of Immunology, vol. 192, no. 10, pp. 4469-4474, 2014.

[8] T. M. Bui, H. L. Wiesolek, and R. Sumagin, "ICAM-1: a master regulator of cellular responses in inflammation, injury resolution, and tumorigenesis," Journal of Leukocyte Biology, vol. 108, no. 3, pp. 787-799, 2020.

[9] R. Rothlein, E. A. Mainolfi, M. Czajkowski, and S. D. Marlin, "A form of circulating ICAM-1 in human serum," The Journal of Immunology, vol. 147, no. 11, pp. 3788-3793, 1991.

[10] V. Marzolla, A. Armani, C. Mammi et al., "Essential role of ICAM-1 in aldosterone-induced atherosclerosis," International Journal of Cardiology, vol. 232, pp. 233-242, 2017.

[11] H. R. Mohammadi, M. S. Khoshnam, and E. Khoshnam, "Effects of different modes of exercise training on body composition and risk factors for cardiovascular disease in middle-aged men," International Journal of Preventive Medicine, vol. 9, no. 1, p. 9, 2018.

[12] H. Y. Jin and T. S. Park, "Role of inflammatory biomarkers in diabetic peripheral neuropathy," Journal of diabetes investigation, vol. 9, no. 5, pp. 1016-1018, 2018.

[13] A.-M. L. Wegeberg, T. Okdahl, T. Fløyel et al., "Circulating inflammatory markers are inversely associated with heart rate variability measures in type 1 diabetes," Mediators of Inflammation, vol. 2020, 10 pages, 2020.

[14] W. Cai, K. Zhang, P. Li et al., "Dysfunction of the neurovascular unit in ischemic stroke and neurodegenerative diseases: an aging effect," Ageing Research Reviews, vol. 34, pp. 77-87, 2017.

[15] W. Eldahshan, S. C. Fagan, and A. Ergul, "Inflammation within the neurovascular unit: focus on microglia for stroke injury and recovery," Pharmacological Research, vol. 147, p. 104349, 2019.

[16] Q. Wang, W. Yang, J. Zhang, Y. Zhao, and Y. Xu, “TREM2 overexpression attenuates cognitive deficits in experimental models of vascular dementia," Neural Plasticity, vol. 2020, Article ID 8834275, 10 pages, 2020.

[17] Q. Wang, Y. Xu, C. Qi, A. Liu, and Y. Zhao, “Association study of serum soluble TREM2 with vascular dementia in Chinese Han population," The International Journal of Neuroscience, vol. 130, no. 7, pp. 708-712, 2020.

[18] T. Yu, Y. Lin, Y. Xu et al., "Repressor Element 1 Silencing Transcription Factor (REST) Governs Microglia- Like BV2 Cell Migration via Progranulin (PGRN)," Neural Plasticity, vol. 2020, Article ID 8855822, 9 pages, 2020.

[19] X. Du, Y. Xu, S. Chen, and M. Fang, "Inhibited CSF1R alleviates ischemia injury via inhibition of microglia M1 polarization and NLRP3 pathway," Neural Plasticity, vol. 2020, Article ID 8825954, 11 pages, 2020.
[20] W. J. Tu, X. Dong, S. J. Zhao, D. G. Yang, and H. Chen, “Prognostic value of plasma neuroendocrine biomarkers in patients with acute ischaemic stroke," Journal of Neuroendocrinology, vol. 25, no. 9, pp. 771-778, 2013.

[21] X. Wang, F. Zhang, W. Ma, D. Feng, J. Zhang, and J. Xu, "Increased levels of serum neuregulin 1 associated with cognitive impairment in vascular dementia," BioMed Research International, vol. 2020, Article ID 6683747, 5 pages, 2020.

[22] Y. Xu, Q. Wang, Z. Qu, J. Yang, X. Zhang, and Y. Zhao, "Protective effect of hyperbaric oxygen therapy on cognitive function in patients with vascular dementia," Cell Transplantation, vol. 28, no. 8, pp. 1071-1075, 2019.

[23] J. Zhang, L. Tang, J. Hu, Y. Wang, and Y. Xu, "Uric acid is associated with cognitive impairment in the elderly patients receiving maintenance hemodialysis-a two-center study," Brain and Behavior: A Cognitive Neuroscience Perspective, vol. 10, no. 3, p. e01542, 2020.

[24] J. L. Banks and C. A. Marotta, "Outcomes validity and reliability of the modified Rankin scale: implications for stroke clinical trials: a literature review and synthesis," Stroke, vol. 38, no. 3, pp. 1091-1096, 2007.

[25] T. Quinn, J. Dawson, and M. Walters, "Dr John Rankin; his life, legacy and the 50th anniversary of the Rankin stroke scale," Scottish Medical Journal, vol. 53, no. 1, pp. 44-47, 2008.

[26] J. P. Broderick, O. Adeoye, and J. Elm, "Evolution of the modified Rankin scale and its use in future stroke trials," Stroke, vol. 48, no. 7, pp. 2007-2012, 2017.

[27] A. Tuttolomondo, D. Di Raimondo, R. di Sciacca, A. Pinto, and G. Licata, "Inflammatory cytokines in acute ischemic stroke," Current Pharmaceutical Design, vol. 14, no. 33, pp. 3574-3589, 2008.

[28] B. W. McColl, S. M. Allan, and N. J. Rothwell, "Systemic infection, inflammation and acute ischemic stroke," Neuroscience, vol. 158, no. 3, pp. 1049-1061, 2009.

[29] T. Dziedzic, "Systemic inflammation as a therapeutic target in acute ischemic stroke," Expert Review of Neurotherapeutics, vol. 15, no. 5, pp. 523-531, 2015.

[30] S. Amaro, F. Jiménez-Altayó, and Á. Chamorro, "Uric acid therapy for vasculoprotection in acute ischemic stroke," Brain circulation, vol. 5, no. 2, pp. 55-61, 2019.

[31] A. Chamorro, A. Meisel, A. M. Planas, X. Urra, D. Van De Beek, and R. Veltkamp, "The immunology of acute stroke," Nature Reviews Neurology, vol. 8, no. 7, pp. 401-410, 2012.

[32] L. Kang, H. Yu, X. Yang et al., "Neutrophil extracellular traps released by neutrophils impair revascularization and vascular remodeling after stroke," Nature Communications, vol. 11, no. 1, p. 2488, 2020.

[33] M. Ucci, P. Di Tomo, F. Tritschler et al., "Anti-inflammatory role of carotenoids in endothelial cells derived from umbilical cord of women affected by gestational diabetes mellitus," Oxidative Medicine and Cellular Longevity, vol. 2019, 11 pages, 2019.

[34] J. Wang, V. Polaki, S. Chen, and J. C. Bihl, "Exercise improves endothelial function associated with alleviated inflammation and oxidative stress of perivascular adipose tissue in type 2 diabetic mice," Oxidative Medicine and Cellular Longevity, vol. 2020, 12 pages, 2020.

[35] R. Lyck and G. Enzmann, "The physiological roles of ICAM-1 and ICAM-2 in neutrophil migration into tissues," Current Opinion in Hematology, vol. 22, no. 1, pp. 53-59, 2015. 
[36] H. Cung, M. J. Aragon, K. Zychowski et al., "Characterization of a novel endothelial biosensor assay reveals increased cumulative serum inflammatory potential in stabilized coronary artery disease patients," Journal of Translational Medicine, vol. 13, no. 1, 2015.

[37] J. Janowska, J. Chudek, M. Olszanecka-Glinianowicz, E. Semik-Grabarczyk, and B. Zahorska-Markiewicz, "Interdependencies among selected pro-inflammatory markers of endothelial dysfunction, C-peptide, anti-inflammatory interleukin-10 and glucose metabolism disturbance in obese women," International Journal of Medical Sciences, vol. 13, no. 7, pp. 490-499, 2016.

[38] R. L. Zhang, M. Chopp, Y. Li et al., "Anti-ICAM-1 antibody reduces ischemic cell damage after transient middle cerebral artery occlusion in the rat," Neurology, vol. 44, no. 9, pp. 1747-1751, 1994

[39] M. P. Bowes, J. A. Zivin, and R. Rothlein, "Monoclonal antibody to the ICAM-1 adhesion site reduces neurological damage in a rabbit cerebral embolism stroke model," Experimental Neurology, vol. 119, no. 2, pp. 215-219, 1993.

[40] G. U. Enzmann, S. Pavlidou, M. Vaas, J. Klohs, and B. Engelhardt, "ICAM-1null C57BL/6 mice are not protected from experimental ischemic stroke," Translational Stroke Research, vol. 9, no. 6, pp. 608-621, 2018.

[41] P. J. Lindsberg, O. Carpen, A. Paetau, M.-L. KarjalainenLindsberg, and M. Kaste, "Endothelial ICAM-1 expression associated with inflammatory cell response in human ischemic stroke," Circulation, vol. 94, no. 5, pp. 939-945, 1996.

[42] V. Supanc, Z. Biloglav, V. B. Kes, and V. Demarin, "Role of cell adhesion molecules in acute ischemic stroke," Annals of Saudi Medicine, vol. 31, no. 4, pp. 365-370, 2011.

[43] Enlimomab Acute Stroke Trial Investigators, "Use of antiICAM-1 therapy in ischemic stroke: Results of the Enlimomab Acute Stroke Trial," Neurology, vol. 57, no. 8, pp. 1428-1434, 2001.

[44] B. N. Wu, J. Wu, D. L. Hao, L. L. Mao, J. Zhang, and T. T. Huang, "High serum sICAM-1 is correlated with cerebral microbleeds and hemorrhagic transformation in ischemic stroke patients," British Journal of Neurosurgery, vol. 32, no. 6, pp. 631-636, 2018. 